\title{
Formation of a penumbra in a decaying sunspot ${ }^{\star}$
}

\author{
R. E. Louis ${ }^{1}$, S. K. Mathew ${ }^{2}$, K. G. Puschmann ${ }^{1}$, C. Beck ${ }^{3}$, and H. Balthasar ${ }^{1}$ \\ 1 Leibniz-Institut für Astrophysik Potsdam (AIP), An der Sternwarte 16, 14482 Potsdam, Germany \\ e-mail: rlouis@aip.de \\ 2 Udaipur Solar Observatory, Physical Research Laboratory, Dewali, Badi Road, 313004 Udaipur, Rajasthan, India \\ 3 National Solar Observatory, Sacramento Peak, 3010 Coronal Loop, Sunspot, New Mexico 88349, USA
}

Received 18 February 2013 / Accepted 12 March 2013

\section{ABSTRACT}

\begin{abstract}
Context. Penumbrae are an important characteristic of sunspots, whose formation is intricately related to the nature of subphotospheric magnetic fields.

Aims. We study the formation of a penumbra in a decaying sunspot and compare its properties with those seen during the development of a proto-spot.

Methods. High-resolution spectropolarimetric observations of active region NOAA 11283 were obtained from the spectro-polarimeter on board Hinode. These were complemented with full-disk filtergrams of continuum intensity, line-of-sight magnetograms, and dopplergrams from the Helioseismic and Magnetic Imager at high cadence.

Results. The formation of a penumbra in the decaying sunspot occurs after the coalescence of the sunspot with a magnetic fragment/pore, which initially formed in the quiet Sun close to an emerging flux region. At first, a smaller set of penumbral filaments develop near the location of the merger with very bright penumbral grains with intensities of $1.2 I_{\mathrm{QS}}$, upflows of $4 \mathrm{~km} \mathrm{~s}{ }^{-1}$, and a lifetime of $10 \mathrm{~h}$. During the decay of these filaments, a larger segment of a penumbra forms at the location of the coalescence. These new filaments are characterized by nearly supersonic downflows of $6.5 \mathrm{~km} \mathrm{~s}^{-1}$ that change to a regular Evershed flow nearly $3 \mathrm{~h} \mathrm{later.}$ Conclusions. The coalescence of the pore with the decaying sunspot provided sufficient magnetic flux for the penumbra to form in the sunspot. The emerging flux region could have played a decisive role in this process because the formation occurred at the location of the merger and not on the opposite side of the sunspot.
\end{abstract}

Key words. sunspots - Sun: photosphere - Sun: surface magnetism - techniques: photometric - techniques: polarimetric

\section{Introduction}

The radially oriented, filamentary penumbra is a distinct property of sunspots that distinguishes them from pores. The formation of a penumbra in a sunspot is quite rapid and grows in segments when the magnetic flux of the proto-spot is about 1$1.5 \times 10^{19} \mathrm{Mx}$ (Leka \& Skumanich 1998). Yang et al. (2003) and Schlichenmaier et al. (2010b) observed that individual penumbral filaments form on time scales of $30 \mathrm{~min}$, and that it takes nearly $4 \mathrm{~h}$ for the penumbra to cover half the umbral circumference. Transient penumbrae, which do not develop into stable penumbral filaments, also form on similar time scales and have been observed in decaying fragments of sunspots (Louis et al. 2012). Recently, Shimizu et al. (2012) reported the existence of an annular zone in the chromosphere around a sunspot, which could be a precursor to penumbral formation. As the penumbra develops, it fills this annular zone, which according to Shimizu et al. (2012) is different from the sunspot moat region.

The formation of the penumbra is immediately followed by the initiation of the Evershed flow (EF) - a nearly horizontal and radial outflow of plasma (Evershed 1909). Consequently, the center- and limb-side penumbra are blue- and redshifted, respectively, when the sunspot is located off the disk center. However, the early stages of penumbral formation and the late stages of decay are sometimes associated with a counter-Evershed flow that has the opposite sign of the EF (Schlichenmaier et al. 2012; Bellot Rubio et al. 2008). While magnetic flux coalesces with

* An animation of the HMI data is available in electronic form at http: //www . aanda.org the proto-spot at one side, the penumbra was seen to form at the opposite side by Schlichenmaier et al. (2010a). The area outside the proto-spot, close to the emerging flux region is characterized by elongated granules and tiny magnetic bipoles (Rezaei et al. 2012). While it is not fully clear why the penumbra forms in the manner it does in a developing sunspot, even less is known about the properties of penumbra formation during sunspot decay. In this study, we show that mature penumbral filaments can form even during the late stages of sunspot decay. In contrast to the findings of Schlichenmaier et al. (2010b), we observe the formation of a penumbra at the location of coalescence between a decaying sunspot and a tiny pore.

\section{Observations}

For this investigation, we employed full-disk solar images from the Helioseismic and Magnetic Imager (HMI; Schou et al. 2012) on board the Solar Dynamics Observatory (SDO; Pesnell et al. 2012) for active region NOAA 11283 observed on 2011 September 4 . These data include $4 \mathrm{k} \times 4 \mathrm{k}$-pixel images of continuum intensity, line-of-sight (LOS) magnetograms, and dopplergrams of the photospheric Fe I line at $617.3 \mathrm{~nm}$ at a cadence of 12 min and a spatial sampling of $0.5 \mathrm{pixel}^{-1}$.

The same active region was also mapped by the Hinode spectropolarimeter (SP, Lites et al. 2001; Ichimoto et al. 2008) on September 4 from 18:20-19:32 UT. The SP recorded the four Stokes profiles of the Fe I lines at $630 \mathrm{~nm}$ with a spectral sampling of $2.15 \mathrm{pm}$. An $18^{\prime \prime} \times 81^{\prime \prime}$ field-of-view (FOV) encompasses a part of the leading sunspot and a decaying follower sunspot that was repeatedly scanned with a pixel size of 0.16 , 

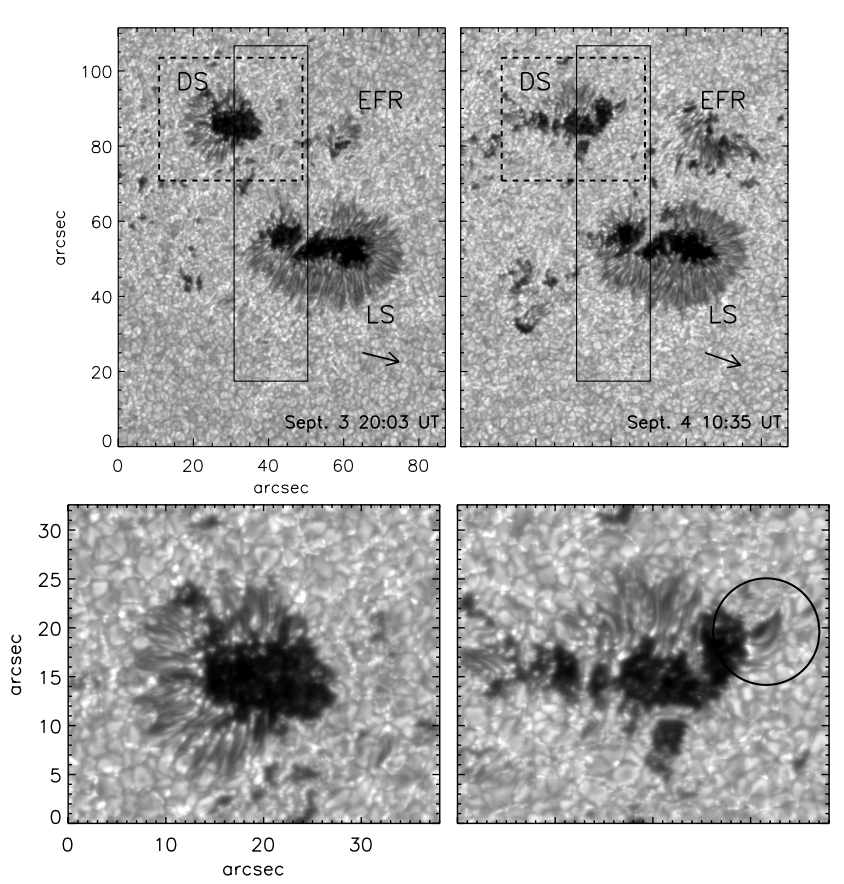

Fig. 1. Active region NOAA 11283. Top: G-band images of the AR on September 3 (left panel) and 4 (right panel). The vertical rectangle corresponds to the field-of-view scanned by the Hinode/SP. LS-leading sunspot, DS-decaying sunspot, and EFR-emerging flux region. The bottom panels correspond to the region outlined by the dashed lines in the top panel. Solar east and north are to the left and to the top.

and an exposure time of $4.8 \mathrm{~s}$ per slit position (normal map mode). The observations were reduced with the corresponding routines of the Solar-Soft package (Lites \& Ichimoto 2013). In $24 \mathrm{~h}$, the active region traversed from a heliocentric angle $(\Theta)$ of $22^{\circ}$ to $\Theta=10^{\circ}$ on September 4 .

\section{Results}

\subsection{Penumbra formation from coalescence}

On 2011 September 4, active region NOAA 11283 consisted of a leading sunspot and a decaying follower spot to its north. The follower sunspot is devoid of a penumbra on its western half (bottom left panel of Fig. 1) close to the end of September 3. There is significant decay in the follower sunspot, nearly $14 \mathrm{~h}$ later, with several light bridges and with the penumbra encircling mostly the northern half of the sunspot. The top panels of Fig. 1 also shows an emerging flux region just outside the decaying sunspot to its west.

We followed the evolution of the decaying sunspot using HMI filtergrams of continuum intensity, dopplergrams, and LOS magnetograms as shown in Fig. 2. The black circle in the top panels indicates an emerging magnetic patch/pore at the beginning of September 4 (continuum image in panel 2), which is located 7 " to the west of the decaying sunspot. The patch is of negative polarity, the same as the decaying sunspot. This patch shows redshifts $(\mathrm{RS})$ of $\approx 1.5 \mathrm{~km} \mathrm{~s}^{-1}$ in the corresponding LOS dopplergram. Panel 4 of Fig. 2 shows the magnetic patch to have increased by a factor of four in area nearly $5 \mathrm{~h}$ after emergence and to have a magnetic flux of $-2.8 \times 10^{19} \mathrm{Mx}$. The patch persistently exhibits strong RS of $\approx 2.5 \mathrm{~km} \mathrm{~s}^{-1}$ and drifts toward the decaying sunspot with a speed of $300 \mathrm{~m} \mathrm{~s}^{-1}$. Nearly $7 \mathrm{~h}$ after emergence, the magnetic patch coalesces with the decaying sunspot (continuum image in panel 5) while continuing to be strongly redshifted. After merging with the decaying sunspot, a part of the magnetic fragment develops into a well-defined penumbral structure (black arrow in panels 5-6 of Fig. 2). A close-up view of this penumbral filament is shown in the lower right panel of Fig. 1, indicated by the black circle. The G-band image also shows traces of rudimentary penumbrae at the umbraquiet Sun boundary south of the bright penumbral grain. While the bright penumbral grain is associated with weak blueshifts (BS) of $470 \mathrm{~m} \mathrm{~s}^{-1}$, the umbra-penumbra boundary immediately south of it continues to exhibit strong RS (dopplergram in panel 6 of Fig. 2). The green arrow in panels 7-9 of Fig. 2 follows the decay of the penumbral filament, and the intensity of the bright penumbral grain exceeds that of the quiet Sun (QS) by $\approx 20 \%$. The lifetime of the transient penumbral filament is $\approx 10 \mathrm{~h}$.

\subsection{Strong redshifts and regular Evershed flow}

While the penumbral filament described in Sect. 3.1 decays, the adjacent region south of it rapidly develops into a distinct penumbral section (maroon arrows in panels $7-10$ of Fig. 2) within $4 \mathrm{~h}$. Prior to its formation, strong RS of $\approx 2.5 \mathrm{~km} \mathrm{~s}^{-1}$ are observed at the umbra-QS boundary (maroon arrow in panel 7). These RS are associated with the magnetic fragment that had earlier merged with the decaying sunspot. Once the penumbra has matured, we detect a regular EF with a blueshift of about $0.7 \mathrm{~km} \mathrm{~s}^{-1}$ in the corresponding dopplergrams (panels 9-10). Time-lapse movies of the continuum intensity images and dopplergrams reveal that even after the initiation of penumbral formation, the strong RS persist for nearly $3 \mathrm{~h}$ before the regular EF starts. The newly formed penumbra shown in panel 9 of Fig. 2 contains a magnetic flux of $\approx-2.0 \times 10^{19} \mathrm{Mx}$ and lies in close proximity to the emerging flux region (top right panel of Fig. 1).

\subsection{Strong velocities in the penumbra}

Figure 3 shows a sequence of Hinode/SP scans of the decaying sunspot when it was at $\Theta=14^{\circ}$. The bisector LOS-velocities shown in the bottom panel were derived at the $70 \%$ intensity level of the Fe $6301.5 \AA$ line. The figure shows the decay of the penumbral filament with the very bright penumbral grain (dashed circle). The continuum images in panels 6-7 clearly show elongated granules that are flanked by dark intergranular lanes that are reminiscent of the decaying penumbral structure. These rudimentary filaments are associated with a blueshift of up to $1.0 \mathrm{~km} \mathrm{~s}^{-1}$ (panel 1), which decrease as the structure decays. In comparison, the larger, well-developed penumbral section includes both the regular EF and strong RS, as seen in the corresponding LOS velocity maps. The EF is associated with a blueshift of nearly $2.0 \mathrm{~km} \mathrm{~s}^{-1}$ while the RS are about $3.5 \mathrm{~km} \mathrm{~s}^{-1}$. The black arrow points to one location of the RS that lies next to the regular EF. The sequence of the SP scans shows that the strong redshifted patch in the penumbra reduces in area, and the velocity decreases to $1.5 \mathrm{~km} \mathrm{~s}^{-1}$ in about $1 \mathrm{~h}$.

Figure 4 shows the Stokes profiles (filled circles) emanating from a pixel in the bright penumbral region (green triangle in panel 1 of Fig. 3) and in the redshifted patch (green square in panel 1 of Fig. 3) in the top and bottom panels, respectively. The top panel indicates that the continuum intensity exceeds that of the quiet Sun by $\approx 20 \%$, which is also seen in the HMI images. In addition, the Stokes $V$ profiles of both lines exhibit a small bump in the blue lobe, which is a characteristic feature of the photospheric EF. The Stokes I profiles corresponding to 


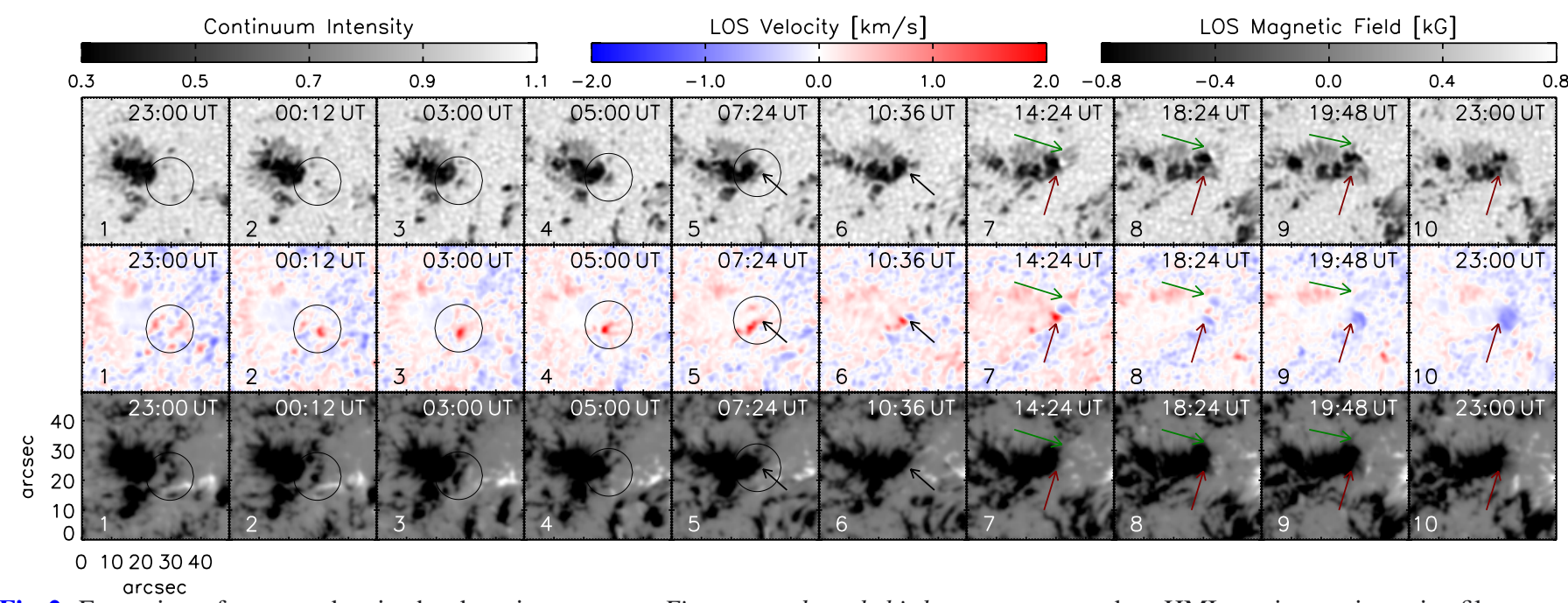

Fig. 2. Formation of a penumbra in the decaying sunspot. First, second, and third rows correspond to HMI continuum intensity filtergrams, dopplergrams, and LOS magnetograms. All images were scaled according to their respective color bars shown above. For the dopplergrams, blue and red signify blueshifts and redshifts. The temporal evolution with a larger field of view covering a longer time span is shown in the movie available in the online edition. In that movie the square indicates the field of view of the panels show in this figure.
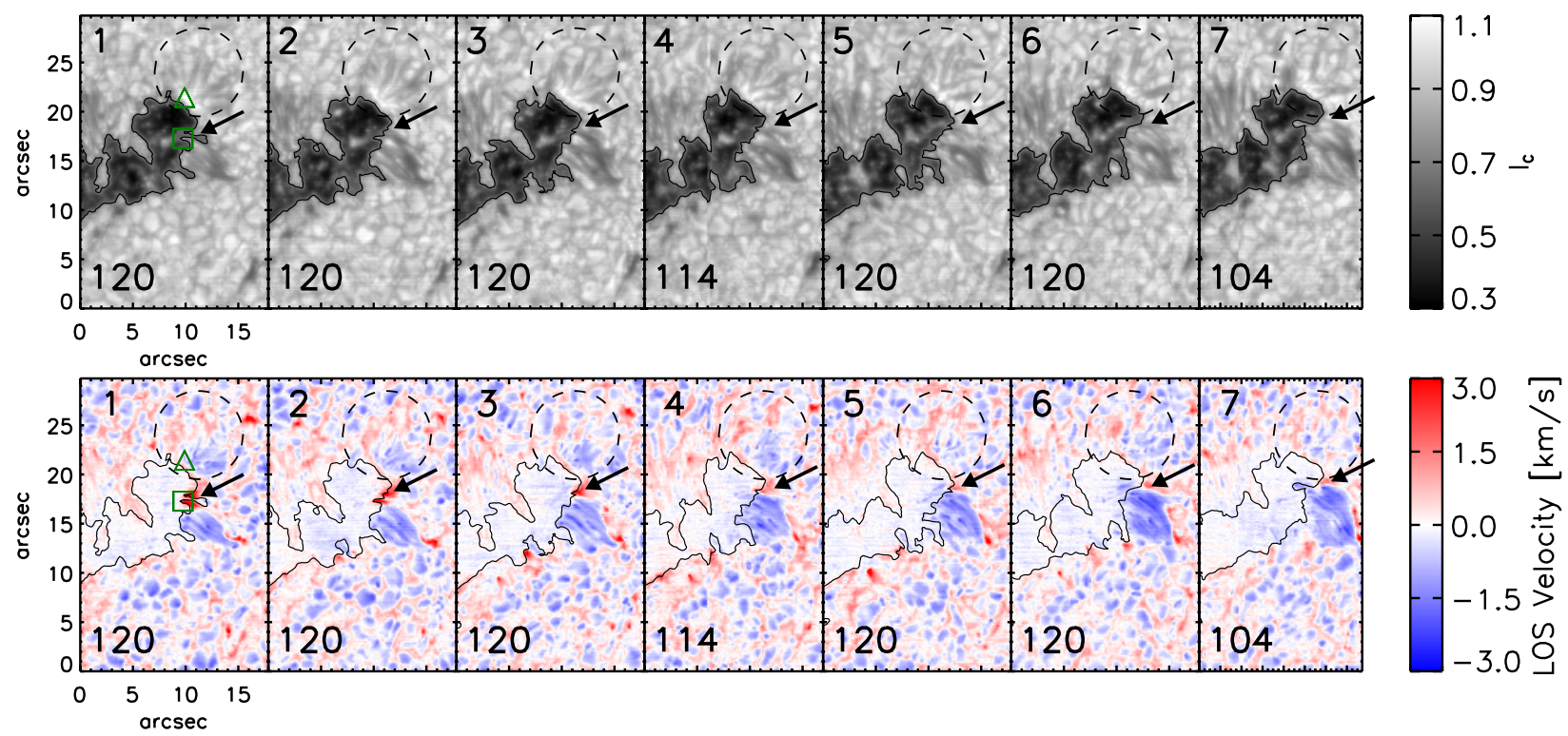

Fig. 3. Regular Evershed flow and strong red-shifts in penumbrae from Hinode/SP observations. Top panels - continuum intensity at $630 \mathrm{~nm}$. Bottom panels - bisector velocity derived at the $70 \%$ intensity level of the $6301.5 \AA$ line. Panel numbers are indicated on the top left corner while the number of scan steps for each panel is shown at the bottom left. The start of the scanning sequence coincides with panel 8 of Fig. 2 . The green triangle and square are centered on pixels whose Stokes profiles are shown in Fig. 4.

the RS (bottom panel of Fig. 4) are characterized by highly inclined red wings, while the $V$ signals show an additional red lobe that signifies a high velocity and has the same polarity as the sunspot. The velocities were estimated by inverting the observed profiles with the SIR code (Stokes Inversion based on Response functions, Ruiz Cobo \& del Toro Iniesta 1992). A two-component model atmosphere was assumed with heightindependent parameters except for the temperature, which was perturbed with two nodes. The observed and inverted profiles are indicated by filled circles and solid lines in Fig. 4. We find that the penumbral filament with the intense brightening at the umbra-penumbra boundary has a BS of $3.8 \mathrm{~km} \mathrm{~s}^{-1}$ in one of the components, whose fill fraction is about $26 \%$. The field strengths and inclinations (relative to the solar surface) for the two components are quite similar with values of about $1.1 \mathrm{kG}$ and $117^{\circ}$. In the redshifted patch, one of the components exhibits near supersonic velocities of about $6.5 \mathrm{~km} \mathrm{~s}^{-1}$, while the other component is at rest. The magnetic field in the redshifted patch is stronger $(2.3 \mathrm{kG})$ and more vertical $\left(135^{\circ}\right)$ in both components than at the location of the intense brigthening. The additional lobe in the red-wing in Stokes $U$, which is not reproduced by the two-component inversion, presumably emanates from a third magnetic component that is nearly horizontal and only weakly $\left(\sim 0.5 \mathrm{~km} \mathrm{~s}^{-1}\right)$ redshifted.

\section{Summary and conclusions}

We have analyzed the process of penumbral formation in a decaying sunspot by combining observations from SDO/HMI and the Hinode/SP. The formation of the penumbra is initiated after a 

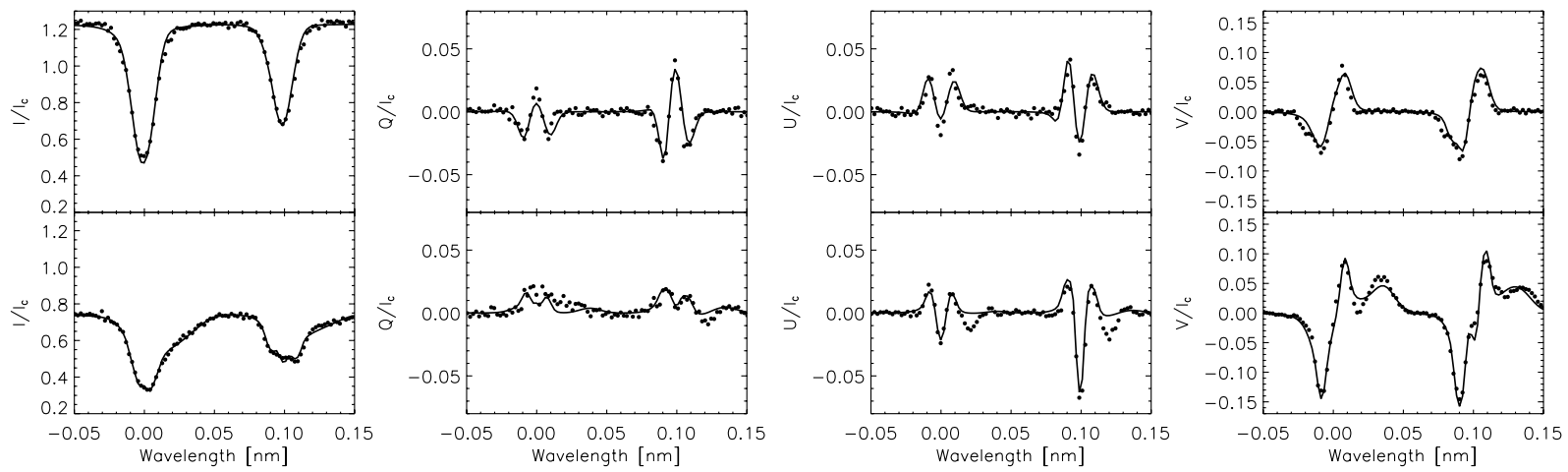

Fig. 4. Stokes profiles corresponding to the bright penumbral region (top panel) and the strong downflows (bottom panel). The observed and inverted profiles are shown by filled circles and solid lines, respectively.

magnetic fragment/pore coalesced with the decaying sunspot in the vicinity of an emerging flux region. This fragment emerged in the QS about 7" to the west of the decaying sunspot. During its motion towards the decaying sunspot, the fragment continuously exhibits redshifts exceeding $2 \mathrm{~km} \mathrm{~s}^{-1}$ that persists even after coalescence. Initially, a small set of penumbral filaments forms at the location of the merger and survives for about $10 \mathrm{~h}$. During the decay of these filaments, the umbra-penumbra boundary is characterized by blueshifts of nearly $4 \mathrm{~km} \mathrm{~s}^{-1}$ and intensities exceeding those of the QS by $20 \%$.

The region south of the decaying penumbral filament rapidly develops into a large penumbral segment with nearly the same magnetic flux as the fragment that had earlier merged with the sunspot. These filaments are initially associated with nearly supersonic redshifts of $6.5 \mathrm{~km} \mathrm{~s}^{-1}$, which change after $3 \mathrm{~h}$ to a regular EF with relatively weak blueshifts of about $2 \mathrm{~km} \mathrm{~s}^{-1}$. Since the sunspot was close to disk center at the time of the SP observations $\left(\Theta=14^{\circ}\right)$, the LOS velocities derived from the bisector method and the inversions practically refer to the vertical component of the plasma motion relative to the solar surface. Therefore, blueshifts and redshifts imply upflows and downflows. A sonic horizontal radial outflow (inflow) would yield an LOS blueshift (redshift) of only about $1.5 \mathrm{~km} \mathrm{~s}^{-1}$ at the heliocentric angle of the observations. The downflows and regular EF are seen to coexist in the penumbra. The EF starts as weak upflows in the inner penumbra (Bellot Rubio et al. 2006; Beck 2008; Franz \& Schlichenmaier 2009; Puschmann et al. 2010) and ends as downflows in the outer penumbra or beyond the sunspot boundary (Westendorp Plaza et al. 1997; Bellot Rubio et al. 2004; Franz \& Schlichenmaier 2013). While the downflows associated with the EF have a polarity opposite to that of the sunspot, the downflows reported here have the same polarity as the decaying sunspot. It is unclear at this point how these strong downflows change to the regular EF and if they have any relation to the supersonic downflows at the umbra-penumbra boundary that are possibly related to reconnection effects (Louis et al. 2011).

The formation of penumbrae in a decaying sunspot is similar to and differs from that observed in a developing proto-spot. Penumbrae form as a result of an increase in magnetic flux, as in the present case, which was supplied by the magnetic fragment/pore that merged with the decaying sunspot. In addition, the penumbra was associated with redshifts during the initial stages of formation, which agrees with Schlichenmaier et al. (2012). However, in contrast to Schlichenmaier et al. (2010b), we found that the penumbra forms at the location of the coalescing fragment, in close proximity to an emerging flux region and not on the opposite side of the sunspot. While the penumbral segment was observed until the end of the September 5, the follower sunspot completely decayed by the end of September 6. The emerging flux region developed into a nearly complete sunspot $48 \mathrm{~h}$ later. These similarities and differences could be attributed to the emerging flux region close to the decaying sunspot, where the growth of penumbrae involves a complex interaction of several individual flux systems in the subphotosphere.

Acknowledgements. Hinode is a Japanese mission developed and launched by ISAS/JAXA, with NAOJ as domestic partner and NASA and STFC (UK) as international partners. It is operated by these agencies in co-operation with ESA and NSC (Norway). HMI data are courtesy of NASA/SDO and the HMI science team. They are provided by the Joint Science Operations Center - Science Data Processing at Stanford University. R.E.L. is grateful for the financial assistance from the German Science Foundation (DFG) under grant DE 787/3-1. R.E.L. appreciates the suggestions from P. Venkatakrishnan and Carsten Denker on the manuscript. We thank the referee Rolf Schlichenmaier for his valuable comments.

\section{References}

Beck, C. 2008, A\&A, 450, 825

Bellot Rubio, L. R., Balthasar, H., \& Collados, M. 2004, A\&A, 427, 319

Bellot Rubio, L. R., Schlichenmaier, R., \& Tritschler, A. 2006, A\&A, 453, 111

Bellot Rubio, L. R., Tritschler, A., \& Martínez Pillet, V. 2008, ApJ, 676, 698

Evershed, J. 1909, MNRAS, 69, 454

Franz, M., \& Schlichenmaier, R. 2009, A\&A, 508, 1453

Franz, M., \& Schlichenmaier, R. 2013, A\&A, 550, A97

Ichimoto, K., Lites, B., Elmore, D., et al. 2008, Sol. Phys., 249, 233

Leka, K. D., \& Skumanich, A. 1998, ApJ, 507, 454

Lites, B. W., \& Ichimoto, K. 2013, Sol. Phys.,

DOI: $10.1007 / \mathrm{s} 11207-012-0205-4$

Lites, B. W., Elmore, D. F., \& Streander, K. V. 2001, in Advanced Solar Polarimetry - Theory, Observation, and Instrumentation, ed. M. Sigwarth, ASP Conf. Ser., 236, 33

Louis, R. E., Bellot Rubio, L. R., Mathew, S. K., \& Venkatakrishnan, P. 2011 , ApJ, 727, 49

Louis, R. E., Ravindra, B., Mathew, S. K., et al. 2012, ApJ, 755, 16

Pesnell, W. D., Thompson, B. J., \& Chamberlin, P. C. 2012, Sol. Phys., 275, 3

Puschmann, K. G., Ruiz Cobo, B., \& Martínez Pillet, V. 2010, ApJ, 720, 1417

Rezaei, R., Bello González, N., \& Schlichenmaier, R. 2012, A\&A, 537, A19

Ruiz Cobo, B., \& del Toro Iniesta, J. C. 1992, ApJ, 398, 375

Schlichenmaier, R., Bello González, N., Rezaei, R., \& Waldmann, T. A. 2010a, Astron. Nachr., 331, 563

Schlichenmaier, R., Rezaei, R., Bello González, N., \& Waldmann, T. A. 2010b, A\&A, 512, L1

Schlichenmaier, R., Rezaei, R., \& Bello González, N. 2012, in 4th Hinode Science Meeting: Unsolved Problems and Recent Insights, eds. L. Bellot Rubio, F. Reale, \& M. Carlsson, ASP Conf. Ser., 455, 61

Schou, J., Scherrer, P. H., Bush, R. I., et al. 2012, Sol. Phys., 275, 229

Shimizu, T., Ichimoto, K., \& Suematsu, Y. 2012, ApJ, 747, L18

Westendorp Plaza, C., del Toro Iniesta, J. C., Ruiz Cobo, B., et al. 1997, Nature, 389,47

Yang, G., Xu, Y., Wang, H., \& Denker, C. 2003, ApJ, 597, 1190 